Revista Brasileira de Cartografia

ISSN 1808-0936 | https://doi.org/10.14393/revbrascartogr

Sociedade Brasileira de Cartografia, Geodésia, Fotogrametria e Sensoriamento Remoto

\title{
Determinação dos Parâmetros de Desalinhamento Angular Usando uma Abordagem Ponto-a-Plano com Apoio de Campo Derivado de Nuvem LiDAR
}

\section{Determination of Boresight Misalignment Parameters using Point-to-Plane Approach with LiDAR point cloud as ground referential}

José Roberto Berithe Pedrosa ${ }^{1}$, Daniel Rodrigues dos Santos ${ }^{2}$

1 Universidade Federal do Paraná, Departamento de Geomática, Curitiba, Brasil. E-mail. pedrosa.jose@eb.mil.br ORCID: https://orcid.org/0000-0001-6268-942X

2 Universidade Federal do Paraná, Departamento de Geomática, Curitiba, Brasil. E-mail. danielsantos@ufpr.br ORCID: https://orcid.org/0000-0001-7977-7426

Resumo: A orientação direta de imagens é uma tarefa essencial para a extração de produtos fotogramétricos com alto grau de confiabilidade. Contudo, para se obter produtos cartográficos com acurácia e precisão posicional desejados é necessário realizar a calibração completa do sistema de mapeamento que envolve a determinação dos parâmetros de orientação interior da câmera e a estimativa dos parâmetros de montagem do sistema. Os parâmetros de montagem são representados pelo braço do sistema, que corresponde ao deslocamento linear entre a origem do sistema referencial INS e o centro perspectivo da câmera, e os parâmetros de orientação relativa, que representam o desalinhamento angular entre o sistema referencial do INS e o sistema referencial fotogramétrico. Este artigo apresenta um método para estimativa dos parâmetros de desalinhamento angular do sistema usando uma abordagem ponto-a-plano e apoio de campo extraído de dados LiDAR. O método proposto foi testado em um sub-bloco composto de seis imagens aéreas, sendo três na direção nordeste, e três na direção sudoeste. Os resultados obtidos mostraram que a abordagem desenvolvida estima, efetivamente, os parâmetros de desalinhamento angular propiciando acurácia posicional na determinação de pontos no espaço objeto.

Palavras-chave: Orientação Direta de Imagens. Abordagem Ponto-a-Plano. Parâmetros de Montagem do Sistema.

\begin{abstract}
The direct orientation of images is an essential task for the extraction of photogrammetric products with a high degree of reliability. However, in order to obtain cartographic products with desired positional accuracy, it is necessary to carry out the calibration of the mapping system, which involves the determination of the camera's internal orientation parameters and the estimation of the system's mounting parameters. The geometric relationship between the sensors (mounting parameters) that are the lever-arm offset between the sensors, and the misalignment (boresight angles) between the INS referential system and the photogrammetric reference system. This work presents a method for estimating the boresight angles of the system using a point-to-plane approach and reference ground control extracted from LiDAR data. The proposed method was tested in a sub-block of images (six images from two opposite flight strips). The obtained results showed that the developed approach effectively estimates misalignment angles, providing positional accuracy in the determination of points in the object space.
\end{abstract}

Keywords: Direct Georeferencing. Point-to-Plane Approach. Boresight Angles.

\section{INTRODUÇÃO}

O problema de calibração dos parâmetros de montagem de sistema fotogramétricos aerotransportados é tema central em aplicações fotogramétricas. Tradicionalmente, o georreferenciamento de imagens é feito de forma indireta utilizando como solução medidas observadas em imagens digitais, pontos de controle correspondentes e técnicas de ajustamento simultâneo de feixes de raios de luz perspectivos. Segundo Habib e Kersting (2010), a partir do advento do GNSS (Global Navigation Sattelite System) integrado ao INS (Inertial Navigation System), a orientação de imagens passou a ser feita de forma direta, onde as informações de posição e atitude da plataforma, fornecidas pela integração GNSS/INS, são utilizadas com maior peso no processo de 
fototriangulação de imagens, juntamente com os pontos de ligação. Com a técnica de georreferenciamento direto de imagens, a reconstrução do espaço objeto é obtida por processo de intersecção fotogramétrica usando os parâmetros de orientação exterior (POEs), fornecidos diretamente pela integração GNSS/INS, os parâmetros de orientação interior (POIs), determinados por um processo de calibração de câmeras, e os parâmetros de montagem do sistema, os quais são o desalinhamento entre o referencial do INS e o referencial da câmera (Boresight) e o deslocamento linear entre estes dois sensores (lever-arm), os quais podem ser obtidos por método de calibração.

Contudo, para se obter produtos cartográficos com acurácia e precisão posicional desejados é necessário realizar a calibração completa do sistema de mapeamento que envolve a determinação dos POIs, bem como a estimativa dos parâmetros de montagem do sistema. Os parâmetros de montagem do sistema fotogramétrico são o braço do sistema (lever-arm offset), que corresponde ao deslocamento linear entre a origem do sistema referencial INS e o centro perspectivo (CP) da câmera, e os parâmetros de orientação relativa (boresight angles), que representam o desalinhamento angular entre o sistema referencial do INS e o sistema referencial fotogramétrico.

As primeiras pesquisas envolvendo métodos de calibração de parâmetros de montagem de sistemas fotogramétricos aerotransportados passaram a ser efetivamente realizadas a partir da década de 1990 com a OEEPE (European Organization for Experimental Photogrammetric Research), a atual EuroSDR (European Spatial Data Research). Segundo Heipke e Jacobsen (2002), a EuroSDR passou a promover experimentos com sensores diretamente orientados utilizando a técnica de georreferenciamento direto e orientação integrada de sensores (ISO), uma vez que a calibração do sistema passou a ser um pré-requisito para se obter informações precisas de posição e orientação a partir de informações oriundas da integração GNSS/INS.

Basicamente, duas abordagens de determinação dos parâmetros de montagem do sistema são encontradas na literatura, isto é, o método por etapas e o método simultâneo. O método por etapas consiste em determinar os parâmetros de montagem do sistema a partir das diferenças existentes entre as informações de posição e orientação do $\mathrm{CP}$ da câmera determinados diretamente pela solução GNSS/INS, e os POEs determinados indiretamente por fototriangulação de imagens (KERSTING, 2011). Já no método simultâneo, os parâmetros de montagem do sistema são estimados no ajustamento de bloco através da orientação integrada de sensores (ISO), utilizando os dados derivados da integração GNSS/INS e apoio de campo (HEIPKE et al., 2002; CRAMER; STALLMANN, 2002; HONKAVAARA et al., 2003; SMITH et al., 2006; YUAN, 2008).

Para ambos os métodos expostos, o modelo de correspondência utilizado na estimativa dos parâmetros de montagem do sistema é baseado na abordagem ponto-a-ponto. Esta estratégia exige a correspondência exata entre pontos conjugados, tornando o processo complexo, uma vez que a coleta dos pontos deve ser feita minuciosamente, porém, nas abordagens em que os pontos de apoio são extraídos da nuvem LiDAR não se pode garantir o estabelecimento de correspondências exatas, devido à natureza irregular e discreta dos dados LiDAR. Por outro lado, quando o ponto de apoio LiDAR é extraído pela interseção de no mínimo três planos de telhados adjacentes é necessário acrescentar as tarefas de segmentação e intersecção de planos. Neste artigo, a estratégia utilizada na correspondência entre o espaço-imagem e o espaço-objeto é ponto-a-plano, ou seja, um ponto na imagem e seu respectivo plano no terreno. Para isso, foram extraídos da nuvem LiDAR planos de telhados, e estes utilizados como apoio de campo. Para testar a precisão deste método, foi feita a interseção fotogramétrica com georreferenciamento direto, e calculado o Erro Médio Quadrático (EMQ) para quinze pontos de verificação.

\section{METODOLOGIA}

Nesta seção, apresenta-se as etapas do método proposto, assim como o modelo matemático utilizado e seu uso para estimar os ângulos de desalinhamento dos parâmetros de montagem do sistema fotogramétrico. O modelo matemático proposto é baseado em uma abordagem ponto-a-plano usando como apoio de campo planos de telhados derivados do sistema LiDAR. Esta modelagem evita correspondência ponto-a-ponto e não exige pontaria na coleta de pontos correspondentes na imagem digital. A Figura 1 mostra as principais etapas do método proposto. 
Figura 1 - Etapas do Método Proposto.

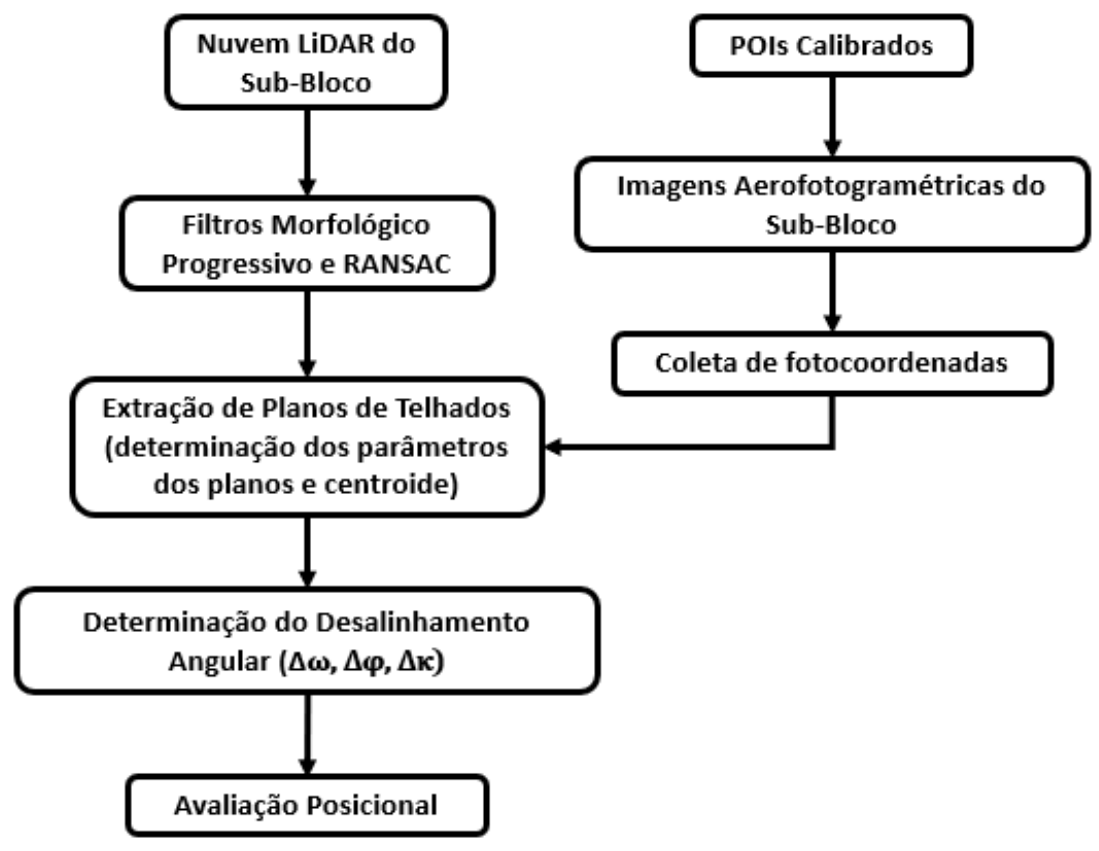

Fonte: Os autores (2020).

Como pode ser observado na Figura 1, os valores dos POIs são obtidos pelo processo de calibração de câmeras in situ, conforme o método de Costa et al.(2017), a nuvem de pontos LiDAR e as fotocoordenadas coletadas no sub-bloco fotogramétrico são usados como dados de entrada no método proposto. Os pontos de elevação são separados dos pontos de terreno e os planos de telhados são extraídos e segmentados. Com isto, os vetores normais dos planos de telhado são estimados e os pontos de controle LiDAR são determinados via intersecção de três planos adjacentes. Finalmente, os parâmetros de desalinhamento angular são estimados usando a abordagem ponto-a-plano proposto neste trabalho e uma avaliação da acurácia posicional é realizado para validação do modelo matemático desenvolvido. A seguir será descrito cada uma das etapas apresentada na Figura 1.

\subsection{Extração automática de planos de telhados na nuvem de pontos LiDAR}

Esta etapa do método consiste em extrair automaticamente planos de telhados e pontos LiDAR presentes na nuvem de pontos. Esta tarefa é dividida em três partes. Primeiro, o Filtro Morfológico Progressivo é executado para separar as elevações (edificações e vegetação) dos pontos de terreno. Em seguida, o algoritmo RANSAC é empregado para extrair superfícies planas na nuvem de pontos LiDAR. Nesta parte do processamento, os pontos que pertencem aos objetos de vegetação são eliminados do processamento uma vez que as seguintes condições para cada ponto da nuvem são estabelecidas como segue:

a) são selecionados os $K$ vizinhos mais próximos do ponto i e aplicado o algoritmo RANSAC. Então, os planos são extraídos com tolerância $\mathcal{E}$ e número $t$ de conjuntos mínimos. O RANSAC extrai o plano que apresenta maior número de pontos;

b) para o ponto i não ser descartado, ele deve atender as seguintes condições: o plano extraído deve possuir um número mínimo de pontos; o ponto i deve pertencer ao plano, ou seja, sua distância em relação ao plano deve ser inferior ao valor da tolerância $\varepsilon$; o ângulo de inclinação do plano em relação à horizontal deve ser superior a $\alpha^{\circ}$ e inferior a $\beta^{\circ}$. Se o ponto i atende essas condições, suas coordenadas são armazenadas, e os parâmetros dos planos são calculados.

Desta forma, os parâmetros do plano $(a, b, c, d)$ são estimados usando o método de Pathak et al. (2010). 
Sabendo que a equação geral do plano é definida como Eq. (1) (STEINBRUCH; WINTERLE, 2006):

$$
a x+b y+c z+d=0
$$

sendo $a, b, c$ e $d$ os parâmetros do plano, $x, y$ e $z$ as coordenadas 3D de um ponto pertencente a superfície plana. A Eq. (1) pode ser reescrita pela multiplicação de duas matrizes, como segue na Eq. (2):

$$
\left[\begin{array}{llll}
x & y & z & 1
\end{array}\right]\left[\begin{array}{l}
a \\
b \\
c \\
d
\end{array}\right]=0
$$

Considera-se que os parâmetros de um plano, extraído na nuvem de pontos LiDAR, são tais que a soma dos quadrados dos resíduos de cada ponto contido neste plano seja minimizada pela Eq. (3).

$$
\sum_{i=1}^{n} \frac{r_{i}^{2}}{\hat{\sigma}_{i}^{2}}=\min
$$

sendo $r_{i}$ o resíduo do plano em relação ao ponto $p_{i}=\left[\begin{array}{lll}x_{i} & y_{i} & z_{i}\end{array}\right]^{T}$ com $i=1,2, \ldots, n$, isto é, $a x_{i}+b y_{i}+$ $c z_{i}+d=r_{i}, n$ é o número de pontos pertencentes ao plano, $\hat{\sigma}_{i}^{2}$ um valor de peso para cada ponto $p_{i}$ dado em função do valor da variância da distância do ponto $p_{i}$ à origem do sistema.

O somatório na Eq. (3) pode ser reescrito como o somatório de uma multiplicação de matrizes, como segue na Eq. (4):

$$
\sum_{i=1}^{n} r_{i}^{2}=\sum_{i=1}^{n}\left(\frac{1}{\hat{\sigma}_{i}^{2}}\left(\left[\begin{array}{llll}
x_{i} & y_{i} & z_{i} & 1
\end{array}\right]\left[\begin{array}{l}
a \\
b \\
c \\
d
\end{array}\right]\right)^{T}\left[\begin{array}{llll}
x_{i} & y_{i} & z_{i} & 1
\end{array}\right]\left[\begin{array}{l}
a \\
b \\
c \\
d
\end{array}\right]\right)=\min
$$

Utilizando as propriedades de multiplicação de matrizes tem-se a representação matricial mostrada na Eq. (5):

$$
\left[\begin{array}{llll}
a & b & c & d
\end{array}\right]\left(\sum_{i=1}^{n} \frac{1}{\hat{\sigma}_{i}^{2}}\left(\left[\begin{array}{c}
x_{i} \\
y_{i} \\
z_{i} \\
1
\end{array}\right]\left[\begin{array}{llll}
x_{i} & y_{i} & z_{i} & 1
\end{array}\right]\right)\left[\begin{array}{l}
a \\
b \\
c \\
d
\end{array}\right]=\left[\begin{array}{llll}
a & b & c & d
\end{array}\right] B\left[\begin{array}{l}
a \\
b \\
c \\
d
\end{array}\right]=\min \right.
$$

Como a matriz $B=\sum_{i=1}^{n}\left(\left[\begin{array}{llll}x_{i} & y_{i} & z_{i} & 1\end{array}\right]^{T}\left[\begin{array}{llll}x_{i} & y_{i} & z_{i} & 1\end{array}\right]\right)$ é uma matriz $4 \times 4$ simétrica, logo essa matriz tem os autovalores reais, $\lambda_{1}, \lambda_{2}, \lambda_{3}, \lambda_{4}$ sendo $\lambda_{1} \geq \lambda_{2} \geq \lambda_{3} \geq \lambda_{4}$ com os correspondentes autovetores $\boldsymbol{v}_{1}, \boldsymbol{v}_{2}, \boldsymbol{v}_{3}, \boldsymbol{v}_{4}$ unitários e ortogonais entre eles. Desta forma, pode-se aplicar o seguinte teorema apresentado por Anton e Rorres (2001), a saber:

Teorema: Seja $B$ uma matriz $n \times n$ simétrica, cujos autovalores em ordem decrescente de tamanho são $\lambda_{1} \geq$ $\lambda_{2} \geq \cdots \geq \lambda_{n}$ e seja o vetor $\boldsymbol{v}$ restrito a $\|\boldsymbol{v}\|=1$, relativamente ao produto interno euclidiano de $\mathbb{R}^{n}$, então:
a) $\lambda_{1} \geq \boldsymbol{v}^{T} \boldsymbol{A} \boldsymbol{v} \geq \lambda_{n}$
b) $\boldsymbol{v}^{T} A \boldsymbol{v}=\lambda_{n}$ se $\boldsymbol{v}$ é um autovetor de $A$ associado a $\lambda_{n}$;
c) $\boldsymbol{v}^{T} A \boldsymbol{v}=\lambda_{1}$ se $\boldsymbol{v}$ é um autovetor de $A$ associado a $\lambda_{1}$. 
Deste modo, os parâmetros do plano são obtidos por meio das componentes do autovetor $\boldsymbol{v}_{4}$, que corresponde ao menor autovalor $\lambda_{4}$ da matriz $B$. Depois desse processo, os parâmetros supracitados precisam ser normalizados de forma que o vetor normal ao plano seja unitário, como segue na Eq. (6):

$$
\left[\begin{array}{l}
a \\
b \\
c \\
d
\end{array}\right]=\frac{1}{\sqrt{1-v_{4_{w}}{ }^{2}}}\left[\begin{array}{l}
v_{4_{x}} \\
v_{4_{y}} \\
v_{4_{z}} \\
v_{4_{w}}
\end{array}\right]
$$

sendo $v_{4_{x}}, v_{4_{y}}$ e $v_{4_{z}}$ as coordenadas do autovetor $v_{4}$, e $v_{4_{w}}$ o termo independente.

Por fim, é obtido um arquivo em formato texto com os parâmetros dos planos numerados, conforme o número de planos extraídos da nuvem LiDAR, as coordenadas do centroide de cada plano e o número de pontos de cada plano extraído. Após esta etapa, são determinados os Pontos de Controle LiDAR (LCPs) através da intersecção de planos de telhados adjacentes. Vale salientar que esta tarefa é feita de forma manual.

\subsection{Estratégia de correspondência ponto-a-plano}

Para cada imagem digital é selecionado, manualmente, pontos imagem correspondentes aos planos de telhados, automaticamente extraídos na nuvem de pontos LiDAR. Em outras palavras, o operador identifica os planos de telhados extraídos na nuvem LiDAR e coleta o ponto imagem no telhado correspondente na imagem digital, como mostra a Figura 2.

Figura 2 - (a) Planos de telhados extraídos da nuvem lidar, (b) Ponto imagem correspondente.

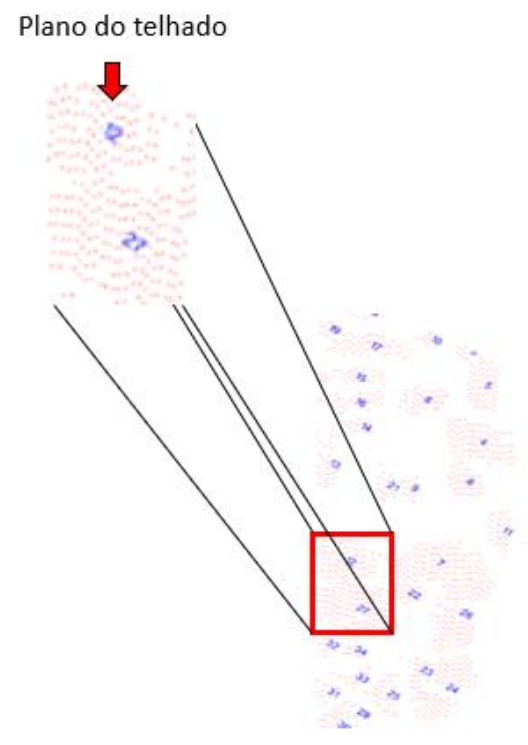

(a)

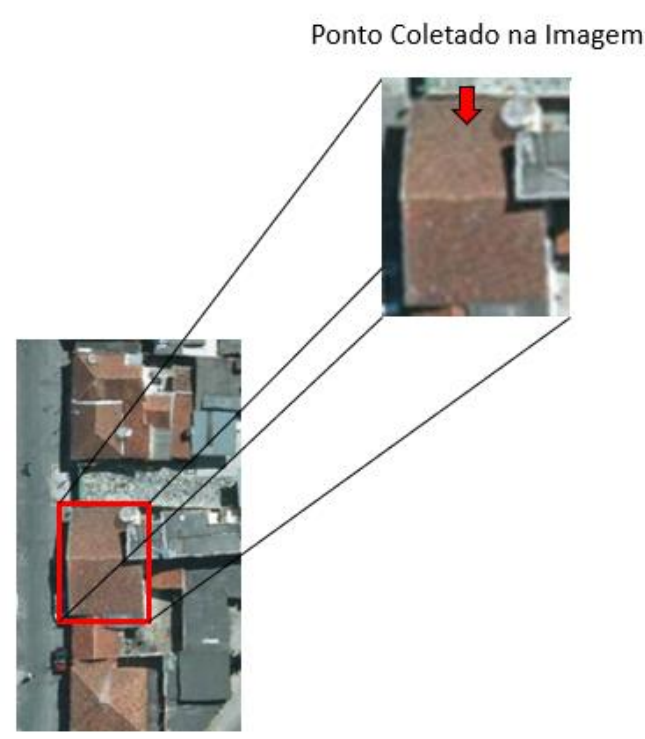

(b)

Fonte: Os autores (2020).

Como pode ser visto na Figura 2a, cada plano de telhado extraído é representado por números. Os planos de telhados são reprojetados para a imagem digital usando os valores dos POIs calibrados, e dos POEs, derivados do INS, apenas para facilitar a identificação do telhado correspondente na imagem digital.

\subsection{Determinação dos parâmetros de desalinhamento angular}

A formulação do modelo matemático proposto neste trabalho é baseada em uma abordagem ponto-a- 
plano. O modelo proposto parte de duas premissas básicas. Primeiro, em situações de georreferenciamento direto de imagens, as coordenadas de um ponto no espaço objeto $\left(r_{I}^{m}\right)$ são determinadas pela soma de três vetores, como segue na Eq. (7):

$$
r_{I}^{m}=r_{b}^{m}(t)+R_{m}^{b}(t) r_{c}^{b}+\lambda_{i} R_{m}^{b}(t) R_{c}^{b} r_{i}^{C}
$$

sendo $r_{b}^{m}(t)$ o vetor das coordenadas 3D da origem do sistema de coordenadas do INS, derivada do processo de integração GNSS/INS levando em consideração a transformação entre a origem do sistema de coordenadas do INS e o centro de fase da antena GNSS, $R_{m}^{b}$ a matriz de rotação que relaciona o sistema de coordenadas terrestre com o sistema de coordenadas do INS (yaw, pitch, roll), $r_{c}^{b}$ e $R_{c}^{b}$ os parâmetros de montagem do sistema que compreendem o deslocamento linear e o desalinhamento angular, respectivamente, entre a origem do sistema de coordenadas do INS e o CP da câmera, $\lambda_{i}$ é o fator de escala, $t$ corresponde ao tempo de aquisição de cada um dos dados e $r_{i}^{C}$ é o vetor das fotocoordenadas no sistema referencial fotogramétrico já corrigidas das distorções das lentes.

Um ponto qualquer $p=\left[\begin{array}{lll}x & y & z\end{array}\right]^{T}$, pertencente a um plano extraído da nuvem de pontos LiDAR $(\aleph)$, pode ser representado matematicamente como o produto interno entre dois vetores, conforme equação do plano Eq. (8):

$$
n p+d=0
$$

sendo, $n=[a, b, c]$

Considerando que $r_{I}^{m}=p$, e utilizando as equações de colinearidade modificada na forma inversa tem-se as Eq. (9), Eq. (10) e Eq. (11), a saber:

$$
\begin{aligned}
& x-X_{0}-\left(r_{11} L_{x}+r_{12} L_{y}+r_{13} L_{z}\right)=\lambda_{i}\left(m_{11} x_{c}^{\prime}+m_{21} y_{c}^{\prime}-m_{31} f\right) \\
& y-Y_{0}-\left(r_{21} L_{x}+r_{22} L_{y}+r_{23} L_{z}\right)=\lambda_{i}\left(m_{12} x_{c}^{\prime}+m_{22} y_{c}^{\prime}-m_{32} f\right) \\
& z-Z_{0}-\left(r_{31} L_{x}+r_{32} L_{y}+r_{33} L_{z}\right)=\lambda_{i}\left(m_{13} x_{c}^{\prime}+m_{23} y_{c}^{\prime}-m_{33} f\right)
\end{aligned}
$$

sendo $x, y, z$ as coordenadas de um ponto extraído da nuvem LiDAR, $L_{x}, L_{y}, L_{z}$ os valores de deslocamento linear entre INS e CP da câmera, $X_{0}, Y_{0}, Z_{0}$ a posição do inercial, obtido pelo georreferenciamento direto do GNSS/INS, $m_{i, j}=R_{m}^{b}(t) * R_{C}^{b}$ corresponde aos elementos da matriz de rotação resultante da multiplicação entre as matrizes de rotação do INS e do desalinhamento angular (boresight), $\lambda$ é o fator de escala, $x_{c}^{\prime}$ e $y_{c}^{\prime}$ as fotocoordenadas no sistema referencial fotogramétrico corrigidas das distorções das lentes e $f$ a distância focal calibrada da câmera.

Dividindo as Eq. (9) e Eq. (10) pela Eq. (11) é eliminado o fator de escala $\lambda_{i}$, como segue:

$$
\begin{aligned}
& x=X_{0}+\left(z-Z_{0}\right)\left(\frac{m_{11}\left(x_{c}^{\prime}+L A_{1}\right)+m_{21}\left(y_{c}^{\prime}+L A_{2}\right)-m_{31}\left(f+L A_{3}\right)}{m_{13}\left(x_{c}^{\prime}+L A_{1}\right)+m_{23}\left(y_{c}^{\prime}+L A_{2}\right)-m_{33}\left(f+L A_{3}\right)}\right) \\
& y=Y_{0}+\left(z-Z_{0}\right)\left(\frac{m_{12}\left(x_{c}^{\prime}+L A_{1}\right)+m_{22}\left(y_{c}^{\prime}+L A_{2}\right)-m_{32}\left(f+L A_{3}\right)}{m_{13}\left(x_{c}^{\prime}+L A_{1}\right)+m_{23}\left(y_{c}^{\prime}+L A_{2}\right)-m_{33}\left(f+L A_{3}\right)}\right)
\end{aligned}
$$

sendo $L A_{1}, L A_{2}, L A_{3}$, é o deslocamento linear (lever-arm) multiplicado pela matriz de rotação do INS $R_{m}^{b}(t)$.

Substituindo as Eq. (12) e Eq. (13) na Eq. (8) tem-se: 


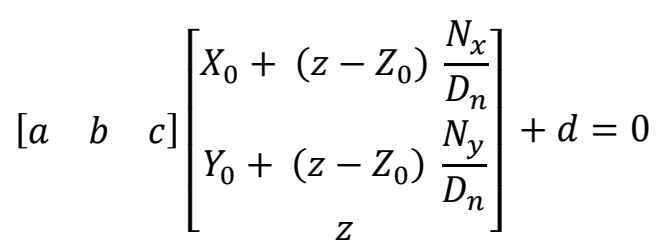

sendo, $a, b, c, d$ os parâmetros do plano, $X_{0}, Y_{0}, Z_{0}$ a posição fornecida pelo GNSS/INS, $z$ a coordenada obtida da nuvem de pontos LiDAR, $N_{x}=m_{11}\left(x_{c}^{\prime}+L A_{1}\right)+m_{21}\left(y_{c}^{\prime}+L A_{2}\right)-m_{31}\left(f+L A_{3}\right), N_{y}=m_{12}\left(x_{c}^{\prime}+\right.$ $\left.L A_{1}\right)+m_{22}\left(y_{c}^{\prime}+L A_{2}\right)-m_{32}\left(f+L A_{3}\right), \quad D_{n}=m_{13}\left(x_{c}^{\prime}+L A_{1}\right)+m_{23}\left(y_{c}^{\prime}+L A_{2}\right)-m_{33}\left(f+L A_{3}\right)$, $x_{c}^{\prime}$ e $y_{c}^{\prime}$ são as fotocoordenadas corrigidas das distorções das lentes, $f$ é a distância focal calibrada $L A_{1}=$ $L_{x} r_{11}+L_{y} r_{21}+L_{z} r_{31}, L A_{2}=L_{x} r_{12}+L_{y} r_{22}+L_{z} r_{32}, L A_{3}=L_{x} r_{13}+L_{y} r_{23}+L_{z} r_{33}$, com $L_{x}, L_{y}, L_{z}$ as componentes em $x$, y e $z$ do lever-arm e $r_{i j}$ os elementos da matriz de rotação do INS. Os $m_{i j}$ correspondem aos elementos da matriz de rotação resultante da multiplicação entre as matrizes de rotação do INS e do boresight angles $m_{11}=r_{11}+r_{12}(-\Delta \kappa)+r_{13} \Delta \varphi, m_{12}=r_{11} \Delta \kappa+r_{12}+r_{13}(-\Delta \omega), m_{13}=r_{11}(-\Delta \varphi)+$ $r_{12} \Delta \omega+r_{13}, \quad m_{21}=r_{21}+r_{22}(-\Delta \kappa)+r_{23} \Delta \varphi, \quad m_{22}=r_{21} \Delta \kappa+r_{22}+r_{23}(-\Delta \omega), \quad m_{23}=r_{21}(-\Delta \varphi)+$ $r_{22} \Delta \omega+r_{23}, \quad m_{31}=r_{31}+r_{32}(-\Delta \kappa)+r_{33} \Delta \varphi, \quad m_{32}=r_{31} \Delta \kappa+r_{32}+r_{33}(-\Delta \omega), \quad m_{33}=r_{31}(-\Delta \varphi)+$ $r_{32} \Delta \omega+r_{33}$.

A Eq. (14) pode ser reescrita na forma da Eq. (15), a saber:

$$
a\left(X_{0}+\left(z-Z_{0}\right) \frac{N_{x}}{D_{n}}\right)+b\left(Y_{0}+\left(z-Z_{0}\right) \frac{N_{y}}{D_{n}}\right)+c z=-d
$$

A Eq. (15) é o modelo matemático proposto neste trabalho para estimativa dos parâmetros de desalinhamento angular. Neste modelo, os parâmetros de desalinhamento angular, $\Delta \omega, \Delta \varphi, \Delta k$, estão inseridos nos termos $N_{x}, N_{y}$ e $D_{n}$, conforme apresentado na Eq. (14). Admitindo que um conjunto finito de pontos foi coletado no espaço imagem que mapeia seu plano correspondente no espaço objeto, extraído na nuvem de pontos LiDAR, o modelo matemático proposto atende a condição $L_{a}=F\left(X_{a}\right)$ devendo os ângulos de desalinhamento do sistema $\left(R_{c}^{b}\right)$ serem estimados pelo Método dos Mínimos Quadrados (MMQ), conforme Eq. (16):

$$
X=-\left(A^{T} P A\right)^{-1}\left(A^{T} P L\right)
$$

sendo $X$ o vetor das correções dos parâmetros $(\Delta \omega, \Delta \varphi, \Delta k), A$ representa a matriz das derivadas parciais em relação aos parâmetros (boresight angles), inseridos nos termos $N_{x}, N_{y} e D_{n}$, conforme apresentado na Eq. (14), $L$ é calculado em função de $L_{o}$ (vetor das observações aproximadas) e $L_{b}$ (vetor das observações) e $P$ é a matriz dos pesos das observações.

Na Eq. (17) a distância da origem do sistema ao plano $(d)$ é considerado como valor de observação. Os valores do desvio-padrão de $d$ é obtido através da propagação de variância covariância da equação $a x+$ $b y+c z=d$, como segue (KHOSHELHAM, 2016):

$$
\sigma_{d}=\sqrt{C_{x}^{2}+C_{y}^{2}+C_{z}^{2}}+\left(\frac{\sigma^{2}}{n_{\text {plan }}}\right)
$$

sendo, $C_{x}, C_{y}, C_{z}$ as coordenadas do centroide do plano, $\sigma^{2}$ a variância nominal das coordenadas dos pontos na nuvem LiDAR e $n_{\text {plan }}$ é o número de pontos contido em cada plano. 


\section{RESULTADOS E DISCUSSÕES}

Neste experimento foi testado o potencial do modelo matemático proposto para estimar os parâmetros de boresight angles. Para avaliar a qualidade da estimativa foram usadas imagens aéreas da cidade de Pouso Alegre - MG/Brasil, adquiridas em maio de 2015, com altura de voo aproximada de $1600 \mathrm{~m}$, sobreposição lateral de $30 \%$, e sobreposição longitudinal de $60 \%$. O sub-bloco fotogramétrico é composto de 6 (seis) imagens, sendo 3 (três) imagens obtidas na direção sudoeste e 3 (três) imagens tomadas na direção nordeste. As imagens foram adquiridas utilizando a câmera digital UltraCam XP (S/N UC-SXp-1-40813045), que é montada com LinosVexcel Apo-Sironar Digital HR 5.6/100mm. A imagem sintética gerada possui cerca de 195,7 Mpx (11310 x 17310) pixels, com o tamanho do pixel pancromático de $6.0 \mu \mathrm{m}$, e um ground sample distance (GSD) próximo a $0,10 \mathrm{~m}$. A aeronave também está embarcada com um INS da Applanix, modelo POS AV 310, que apresenta as seguintes precisões absolutas pós-processadas (RMS): Posição horizontal de 0,02m e posição vertical de $0,05 \mathrm{~m}$, velocidade $0,01 \frac{\mathrm{m}}{\mathrm{s}}$, roll e pitch $<0,015^{\circ}$ e heading $<0,035^{\circ}$ e um sensor LiDAR aerotransportado da marca Leica ALS60, modelo CUS6 IMU, que possui as seguintes precisões absolutas: Posicional, entre 5 e $30 \mathrm{~cm}$, roll e pitch $<0,0025^{\circ}$, heading $<0,005^{\circ}$. A nuvem de pontos possui densidade aproximada de 5 pontos $/ \mathrm{m}^{2}$.

Como descrito anteriormente, os POIs foram obtidos por Costa et al. (2017). Os autores empregaram o método in situ usando 6 imagens aéreas, sendo 3 na direção de voo nordeste e 3 na direção de voo sudoeste em cada direção de voo, e três pontos LiDAR de controle. A Figura 3 mostra a configuração do bloco e do sub-bloco fotogramétrico usado no processo de calibração da câmera UltraCam XP, os pontos de controle coletados no sub-bloco fotogramétrico (representados na cor verde) e a distribuição dos pontos de verificação, também extraídos da nuvem LiDAR.

Figura 3 - Configuração do sub-bloco e pontos de verificação coletados na área de estudo.

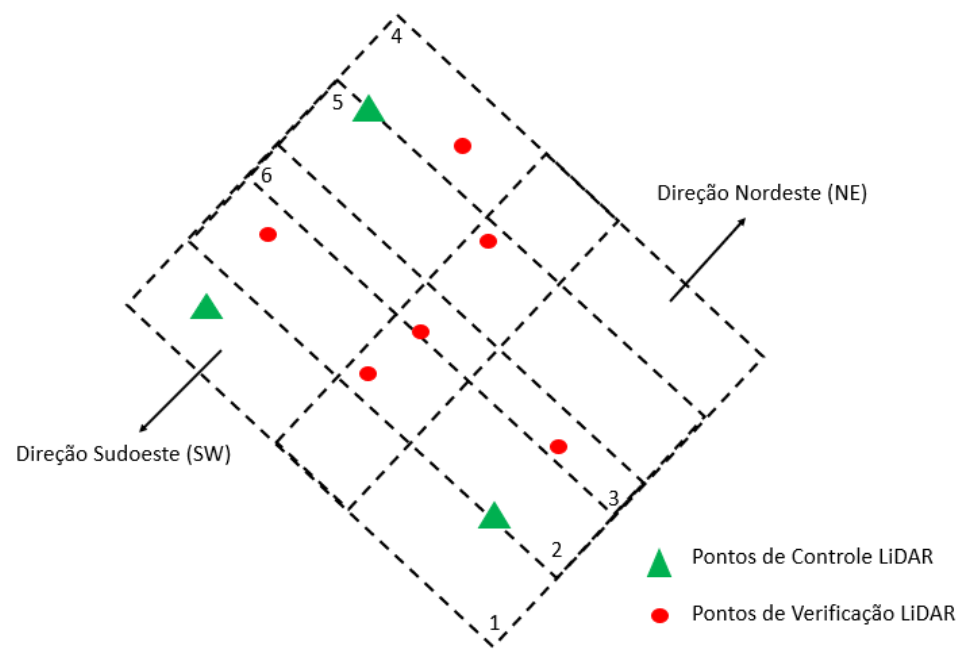

Fonte: Os autores (2020).

Na primeira etapa do método proposto a nuvem de pontos LiDAR é filtrada separando os pontos que estão no nível de solo, dos pontos pertencentes a árvores e edificações. Isto é feito pelo Filtro Morfológico Progressivo. Em seguida, o algoritmo RANSAC é usado para extrair os planos de telhados na nuvem de pontos LiDAR filtrada. Este procedimento está implementado na biblioteca point cloud (PCL), como pode ser verificado em Rusu e Cousins (2011). A Figura 4 mostra a nuvem de pontos LiDAR original, a nuvem filtrada pelo Filtro Morfológico Progressivo e os planos de telhados extraídos com o RANSAC. 
Figura 4 - Resultados da filtragem da nuvem de pontos LiDAR. (a) Nuvem de pontos LiDAR original, (b) Resultado obtido com o filtro morfológico progressivo, (c) Planos de telhados extraídos com o algoritmo RANSAC, (d) Imagem aproximada dos planos extraídos.

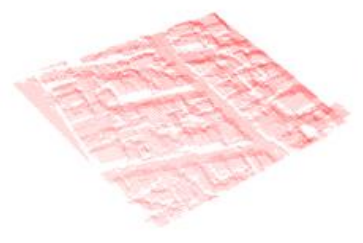

(a)

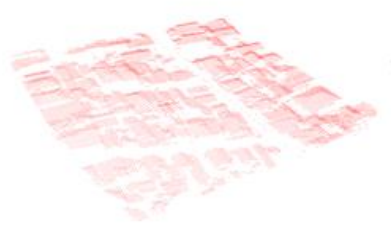

(b)

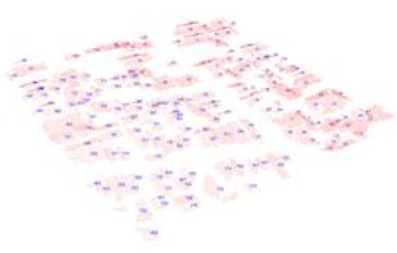

(c)

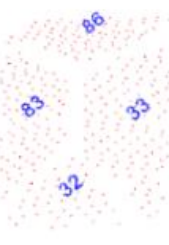

(d)

Fonte: Os autores (2020).

Após aplicar o algoritmo RANSAC, os parâmetros dos planos de telhado são estimados usando o método de Pathak et al. (2010). Para cada plano de telhado extraído na nuvem de pontos LiDAR foi manualmente coletado um ponto qualquer do telhado correspondente na imagem digital. Nesta etapa, foram extraídos 76 (setenta e seis) planos e 15 (quinze) LCPs.

Neste trabalho, o ajustamento por feixes de raios de luz perspectivo é realizado usando 6 imagens do sub-bloco fotogramétrico separados pelas faixas de voo, sendo 3 imagens na direção nordeste do alinhamento de voo e 3 imagens aéreas tomadas na direção sudoeste da trajetória de voo, baseado na pesquisa de Costa (2017). A Figura 5 mostra o sub-bloco fotogramétrico, bem como a distribuição dos pontos de verificação e dos planos de telhados usados como controle de campo.

Figura 5 - Sub-bloco fotogramétrico usado no experimento. (a) configuração dos LCPs no sub-blocos nos sentidos sudoeste e nordeste, (b) distribuição dos planos de telhado.

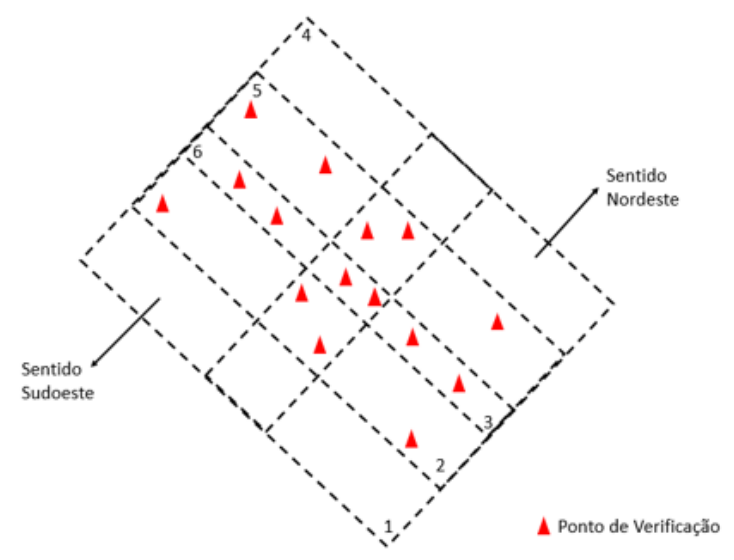

(a)

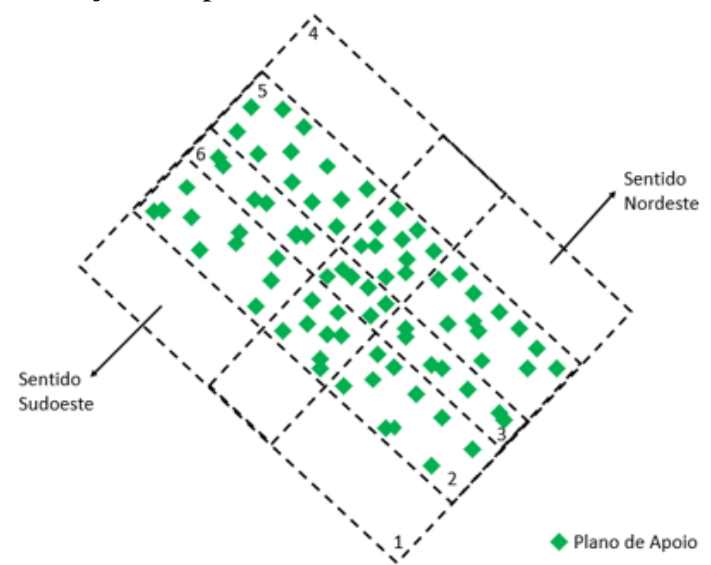

(b)

Fonte: Os autores (2020).

Note que, neste trabalho para cada sentido de voo é determinado um conjunto de parâmetros de boresight angles, ou seja, há 6 parâmetros de desalinhamento a serem determinados, sendo três parâmetros no sentido sudoeste $\left(\Delta \omega_{S O}, \Delta \varphi_{S O}, \Delta \kappa_{S O}\right)$ e três parâmetros no sentido nordeste $\left(\Delta \omega_{N E}, \Delta \varphi_{N E}, \Delta \kappa_{N E}\right)$. A Tabela 1 apresenta os valores de boresight angles e seus desvios-padrão estimados para cada sentido de voo.

Tabela 1 - Resultados obtidos com o método proposto.

\begin{tabular}{|l|l|l|l|l|}
$\Delta$ do desalinhamento & Direção Nordeste & Desvios-padrão & Direção Sudoeste & Desvios-padrão
\end{tabular}




\begin{tabular}{|c|c|c|c|c|}
\hline$\Delta \omega$ & $4^{\prime} 26,43^{\prime \prime}$ & 1'24,98', & $17^{\prime} 18,12^{\prime \prime}$ & $1^{\prime} 12,39^{\prime \prime}$ \\
\hline$\Delta \varphi$ & $7^{\prime} 5,88^{\prime}$ & $1^{\prime} 13,84^{\prime \prime}$ & $-7^{\prime} 48,51^{\prime \prime}$ & $1^{\prime} 18,17$ ' \\
\hline$\Delta \kappa$ & $17,98^{\prime \prime}$ & $5^{\prime} 36,83^{\prime \prime}$ & - 4' 40,96' & $1^{\prime} 18,17^{\prime \prime}$ \\
\hline
\end{tabular}

Fonte: Os autores (2020).

Para integração de dados ópticos e LiDAR é preciso avaliar a qualidade dos valores de boresight angles estimados com o modelo proposto. Neste trabalho, foram extraídos 15 pontos de verificação no referencial de mapeamento usando intersecção de três planos na nuvem de pontos LiDAR. Como a precisão posicional planimétrica dos pontos de verificação pode variar de 0,1 a 1,0 m (VOSSELMAN; MAAS, 2010) será adotado o valor da precisão posicional do EMQ das discrepâncias $(x, y)$ igual a $0,40 \mathrm{~m}$ (duas vezes o desvio-padrão do equipamento LiDAR), e para a precisão altimétrica será adotado o EMQ das discrepâncias (z) igual a 0,48 m, conforme Alamús e Wolfgang (2008):

$$
d_{Z}=\sqrt{2} \cdot \frac{H^{2}}{B \cdot f} \cdot \sigma_{p i x}
$$

sendo $H$ o valor médio da altura de voo, $B$ o valor médio da aerobase, $f$ a distância focal e $\sigma_{\text {pix }}$ o tamanho do pixel. A Tabela 2 mostra os valores adotados para as variáveis supracitadas.

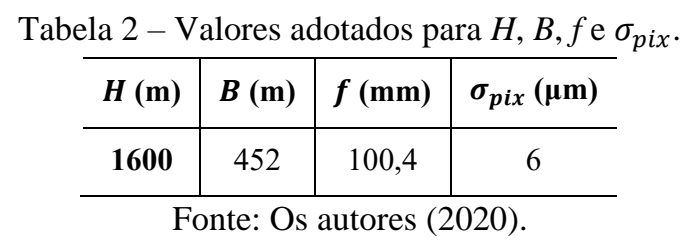

Para a avaliação estatística das discrepâncias das componentes $x, y$ e $z$ resultantes dos pontos de verificação é usado o teste de normalidade de Shapiro-Wilk $(\sigma=0,05)$ e para verificar a existência de tendência nas discrepâncias é empregado o teste $t$ de Student $(\sigma=0,05)$. Os valores de EMQ da discrepância dos pontos de verificação foram de $0,274 \mathrm{~m}$ para planimetria e $0,335 \mathrm{~m}$ para a altimetria, considerando a direção nordeste do alinhamento da faixa de voo. Já para o alinhamento da faixa na direção sudoeste obteve-se um EMQ da discrepância dos pontos de 0,340 m para planimetria e 0,331 $\mathrm{m}$ para a altimetria. Como pode ser observado, os valores obtidos estão dentro do limiar adotado de precisão para as discrepâncias planimétricas e altimétricas. Pode-se observar que o desvio-padrão calculado para o ângulo de desalinhamento $\Delta \kappa$ para o sentido nordeste é maior do que o valor do desalinhamento. A Tabela 3 apresenta os resultados do teste de normalidade de Shapiro-Wilk (S-W), com valores retirados da tabela $\mathrm{S}-\mathrm{W}$ para o sentido de voo nordeste, utilizando oito LCPs, e $\alpha$ com valor de $0,05, W_{(8 ; 0,05)}=0,818$, e para o sentido de voo sudoeste, com sete LCPs, e $\alpha$ com valor de $0,05, W_{(7 ; 0,05)}=0,803$. Como pode ser observado, $W_{\text {calc. }}>W_{\text {tabela }}$ para as três componentes analisadas, com isso, conclui-se que as discrepâncias para as componentes $X, Y$ e $Z$ possuem uma distribuição normal.

Tabela 3 - Resultados do teste de normalidade shapiro-wilk para o experimento b para a direção nordeste (NE) e sudoeste (SO).

\begin{tabular}{c|c|c|c|c|c}
\hline \multicolumn{7}{c|}{ NORDESTE } & \multicolumn{2}{c}{$z$} \\
\hline$W_{\text {calc. }}$ & $\mathrm{p}$-valor & $W_{\text {calc. }}$ & $\mathrm{p}$-valor & $W_{\text {calc. }}$ & $\mathrm{p}$-valor \\
\hline 0,961 & 0,820 & 0,942 & 0,641 & 0,904 & 0,819 \\
\hline \multicolumn{7}{c|}{ SUDOESTE } & \multicolumn{3}{c}{$z$} \\
\hline \multicolumn{2}{c|}{$x$} & $W_{\text {calc. }} y$ & $\mathrm{p}$-valor & $W_{\text {calc. }}$ & $\mathrm{p}$-valor \\
\hline$W_{\text {calc. }}$ & $\mathrm{p}$-valor & 0,869 & 0,188 & 0,867 & 0,183 \\
\hline 0,866 & 0,179 &
\end{tabular}

Fonte: Os autores (2020).

A Figura 6(a) apresenta a discrepância planimétrica e altimétrica dos oito pontos de verificação 
utilizados para a análise estatística para a faixa de voo no sentido nordeste. Nota-se que apenas uma discrepância ultrapassou o limiar de precisão adotado para planimetria, que foi de $0,53 \mathrm{~m}$. Já para a altimetria foi obtido um valor de $0,50 \mathrm{~m}$, ultrapassando o limiar de precisão adotado para discrepância altimétrica. A Figura 6(b) apresenta as discrepâncias planimétrica e altimétrica dos sete pontos LCPs de verificação utilizados para a análise estatística para a faixa de voo no sentido sudoeste. Nota-se que dois pontos ultrapassaram o limiar de precisão adotado para discrepância planimétrica, que foram de $0,56 \mathrm{~m}$ e $0,49 \mathrm{~m}$, enquanto para a altimetria todos os pontos permaneceram dentro do limiar de precisão adotado para discrepância.

Figura 6 - discrepância (a) sentido nordeste e (b) sentido sudoeste.

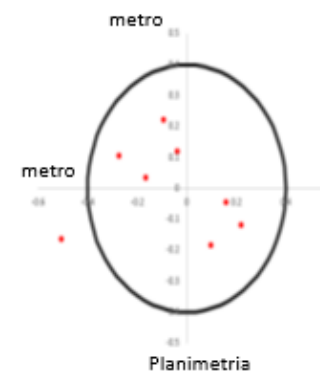

(a)

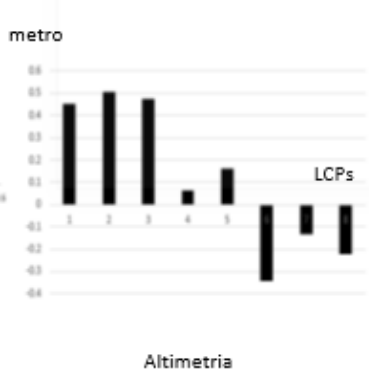

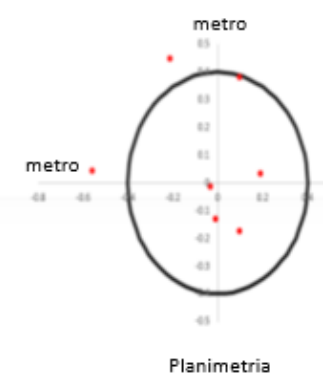

Planimetria



(b)

Fonte: Os autores (2020).

A Tabela 4 apresenta os resultados do teste de tendência $t$ de Student com nível de significância $(\alpha)$ de 5\% para avaliação estatística das discrepâncias da planimetria e da altimetria dos pontos de verificação para as direções de voo nordeste e sudoeste, respectivamente.

Tabela 4 - Resultados do teste estatístico $t$ de Student.

\begin{tabular}{c|c|c|c|c|c|c|c|c}
\hline \multicolumn{2}{c|}{$x$} & \multicolumn{3}{c}{$y$} & \multicolumn{3}{c}{$z$} \\
\hline p-valor & $t_{\text {calc }}$ & $t_{\frac{\alpha}{2}, n-1}$ & $\mathrm{p}$-valor & $t_{\text {calc }}$ & $t_{\frac{\alpha}{2}, n-1}$ & $\mathrm{p}$-valor & $t_{\text {calc }}$ & $t_{\frac{\alpha}{2}, n-1}$ \\
\hline 0,401 & $-0,892$ & 2,145 & 0,954 & $-0,058$ & 2,145 & 0,345 & 1,010 & 2,145 \\
\hline \multicolumn{7}{|c|}{ SUDOESTE } \\
\hline p-valor & $t_{\text {calc }}$ & $t_{\frac{\alpha}{2}, n-1}$ & p-valor & $t_{\text {calc }}$ & $t_{\frac{\alpha}{2}, n-1}$ & p-valor & $t_{\text {calc }}$ & $t_{\frac{\alpha}{2}, n-1}$ \\
\hline 0,543 & $-0,643$ & 2,145 & 0,385 & 0,935 & 2,145 & 0,611 & $-0,535$ & 2,145 \\
\hline
\end{tabular}

Como pode ser observado na Tabela 4 , tanto na planimetria quanto na altimetria foi obtido o p-valor maior que 0,05 e valores absolutos de $t_{c a l c}$ menores que $t_{\frac{\alpha}{2}, n-1}$. Desta forma, pode-se dizer que os pontos não possuem tendência, o que valida os resultados obtidos do EMQ dentro do limiar de precisão adotado. A Tabela 5 apresenta uma síntese dos resultados das discrepâncias dos pontos de verificação obtidos nos experimentos A e B.

Tabela 5 - Síntese dos resultados.

\begin{tabular}{c|c|c|c}
\hline \multicolumn{2}{c|}{ EXPERIMENTO } & Planimetria (m) & Altimetria (m) \\
\hline \multirow{2}{*}{$\begin{array}{c}\text { Resultados para o sentido } \\
\text { nordeste de voo }\end{array}$} & Valor Mínimo & 0,125 & 0,062 \\
\cline { 2 - 4 } & EMQ & 0,274 & 0,335 \\
\cline { 2 - 4 } & Valor Máximo & 0,531 & 0,504 \\
\hline \multirow{2}{*}{$\begin{array}{c}\text { Resultados para o sentido } \\
\text { sudoeste de voo }\end{array}$} & Valor Mínimo & 0,036 & 0,154 \\
\cline { 2 - 4 } & EMQ & 0,340 & 0,331 \\
\cline { 2 - 4 } & Valor Máximo & 0,560 & 0,440 \\
\hline
\end{tabular}

Fonte: Os autores (2020). 
Como pode ser observado na Tabela 5, o EMQ planimétrico foi de $0,274 \mathrm{~m}$ para a faixa de voo no sentido nordeste e o EMQ altimétrico foi de $0,335 \mathrm{~m}$, enquanto para a faixa de voo no sentido sudoeste, o resultado do EMQ planimétrico foi de $0,340 \mathrm{~m}$ e altimétrico de $0,331 \mathrm{~m}$. Com os resultados obtidos, nota-se a foi possível encontrar melhor grau de confiabilidade planialtimétrica utilizando um sub-bloco separado por faixas de voo.

\section{CONCLUSÕES E RECOMENDAÇÕES}

Um dos desafios em sistemas modernos de mapeamento móvel é o fluxo de trabalho na integração de dados fotogramétricos e LiDAR. Nas últimas décadas, a orientação direta de sensores tem ganhado espaço na comunidade fotogramétrica devido sua eficiência e simplicidade operacional. Métodos capazes de determinar os parâmetros de montagem de sistemas aerotransportados é uma área ativa de pesquisa e desenvolvimento. Neste trabalho foi proposto um método apropriado para estimativa dos parâmetros de boresight angles de montagem de um sistema fotogramétrico aerotransportado. A principal característica do método proposto é o desenvolvimento de um modelo matemático baseado em uma abordagem ponto-a-plano que mapeia um ponto na imagem digital em seu plano de telhado correspondente na nuvem de pontos LiDAR aerotransportado. $\mathrm{O}$ desenvolvimento de tal modelo foi motivado pela alta demanda por dados fotogramétricos e LiDAR integrados com acurácia que pode ser empregado em diversas aplicações em geociências. O modelo proposto foi testado em um sub-bloco de imagens aéreas digitais e os resultados obtidos mostraram que planos de telhados podem servir como apoio de campo no processo de estimativa dos parâmetros de boresight angles. O modelo desenvolvido é pela primeira vez aplicado para estimativa dos parâmetros de boresight angles em sistemas aerotransportados. Sua geometria não exige correspondência exata entre as primitivas, descartando necessidade de pontaria na coleta dos pontos no espaço imagem, resultando em um mais flexível método. Superfícies planas são robustas à outliers presentes na nuvem de pontos, além de ser menos influenciadas pela baixa amostragem de pontos e não dependem de pré-sinalização de alvos ou intersecção de planos adjacentes para extração de pontos LiDAR. Como trabalhos futuros é recomentado a utilização deste método aplicado em outro bloco fotogramétrico, assim como a automatização da coleta do ponto na imagem e seu respectivo plano extraído da nuvem LiDAR.

\section{Agradecimentos}

O autor do trabalho externa seus agradecimentos à Diretoria de Serviço Geográfico (DSG) pelo apoio concedido, e ao Instituto LATEC e à empresa TOPOCART por terem cedido os dados na pesquisa.

\section{Contribuição dos autores}

Este artigo foi desenvolvido a partir da pesquisa realizada no mestrado acadêmico do autor principal, sob orientação do coautor. $\mathrm{O}$ autor implementou o algoritmo e realizou os testes com dados reais. O coautor deste trabalho, desenvolveu o modelo matemático e contribuiu com a realização de testes e correções finais do texto.

\section{Conflitos de interesse}

Os autores declaram que não há conflito de interesses. 


\section{Referências}

ANTON, H.; RORRES, C. Álgebra linear com aplicações. Bookman, 8 ed., 2001.

ALAMÚS, R.; WOLFGANG, K. DMC geometry analysis and virtual image characterisation. The Photogrammetric Record, vol.23, $\mathrm{n}^{\circ} 124,353-371,2008$. DOI. 10.1111/j.1477-9730.2008.00504.x

COSTA, F.A.L.; MITISHITA, E.A.; CENTENO, J.A.S. A study of integration of LIDAR and photogrammetric data sets by indirect georeferencing and in situ camera calibration. International Journal of Image and Data Fusion, vol.8, n², 94 - 111, 2017. DOI. 10.1080/19479832.2017.1294625

CRAMER, M., D. STALLMANN. System Calibration for Direct Georeferencing, Remote Sensing and Spatial Information Sciences, vol.3, 479-84, 2002.

GNEENISS, A.. Integration of LiDAR and Photogrammetric Data for Enhanced Aerial Triangulation and Camera Calibration. 2014. 221 f.. Tese em Engenharia civil e Geociências. Newcastle University, Inglaterra, 2013.

HABIB, A.; KERSTING, A. P.. Analysis of Control and Flight Configuration Requirements for Mounting Parameters Calibration of GPS / INS-Assisted Photogrammetric Systems. ISPRS Journal of Photogrammetry and Remote Sensing, vol. 38, 2010, pp. 166-186, Jun. 2010.

HEIPKE, C., JACOBSEN K., WEGMANN H.. Analysis of the Results of the OEEPE Test "Integrated Sensor Orientation", Integrated Sensor Orientation, Test Report and Workshop Proceedings. OEEPE Official Publication, vol.1, $n^{\circ} .43$, p. 31-49, 2002. DOI. 10.1.1.495.4680

HONKAVARA, E., ILVES R., JAAKKOLA, J. Proceedings of International Workshop: Theory, Technology and Realities of Inertial/GPS Sensor Orientation, setembro, 2003, Castelldefels, Spain. Practical results of GPS/IMU camera system calibration, ISPRS, WG I/5, Barcelona, 2003, p. 10-20.

JACOBSEN, K.. Direct/ Integrated Sensor Orientation - Pros and Cons, The International Archives of Photogrammetry and Remote Sensing, vol.35 (Part B3), p. 829-835, 2004.

KERSTING, A. P. B. Quality Assurance of Multi-Sensor Systems, 2011, 295 f. Tese (Doctor of Philosophy) - Department of Geomatics Engineering. University of Calgary, Calgary, 2011.

KHOSHELHAM, K. Closed-form solutions for estimating a rigid motion from plane correspondences extracted from point clouds. ISPRS Journal of Photogrammetry and Remote Sensing, vol. 114, p. 78-91, 2016. DOI. 10.1016/j.isprsjprs.2016.01.010

MITISHITA E., COSTA F., MARLO M.. Study of the Integration of LiDAR and Photogrammetric Datasets by In Situ Camera Calibration and Integrated Sensor Orientation. ISPRS - International Archives of the Photogrammetry, Remote Sensing and Spatial Information Sciences, vol. 42, p. 647-652, 2017. DOI. 10.5194/isprs-archives-XLII-1-W1-647-2017

PATHAK K., BIRK A., VASKEVICIUS N., POPPINGA J. Fast Registration Based on Noisy Planes With Unknown Correspondences for 3-D Mapping. IEEE Transactions on Robotics, vol. 26, no 3, p. 424441, 2010. DOI. 10.1109/TRO.2010.2042989

RUSU, R. B. AND COUSINS, S. 3D is here: Point cloud library (PCL), IEEE International Conference on Robotics and Automation, Shanghai, China, p. 1-4, 2011. DOI. 10.1109/ICRA.2011.5980567

SMITH, M. J., K. S. QTAISHAT, D.W.G. PARK, A. JAMIESON. IMU and Digital Aerial Camera Misalignment Calibration, International Calibration and Orientation Workshop (EuroCOW), Castelldefels, Spain, 2006.

THOM C., Y. RABOT. Study Of Lever-Arm Effect Using Embedded Photogrammetry And On-Board Gps Receiver On Uav For Metrological Mapping Purpose And Proposal Of A Free Ground Measurements Calibration Procedure. The International Archives of the Photogrammetry, Remote Sensing and Spatial Information Sciences, Vol. XL-3/W4, 2016. DOI. 10.5194/isprs-archives-XL-3-W4-652016WEGMANN, H.. Image Orientation by Combined (A) AT with GPS and IMU, ISPRS Com. I, Midterm Symposium, Integrated Remote Sensing at the Global, Denver, U.S.A., p. 10-15, 2002.

YASTIKLI, N., JACOBSEN K. Direct Sensor Orientation for Large Scale Mapping-Potential, Problems, and Solutions. The Photogrammetric Record, vol. 20, nº111, p. 274-284, 2005. DOI. 10.1111/j.14779730.2005.00318.x 
YUAN, X.. A novel method of systematic error compensation for a position and orientation system, Progress in Natural Science, vol. 18, $\mathrm{n}^{\circ}$ 8, p. 953-963, 2008. DOI. 10.1016/j.pnsc.2008.02.005

ZHANG K; CHEN S.C.; WHITMAN D.; SHYU M.L.; YAN, J.; ZHANG, C.A. Progressive Morphological filter for removing nonground measurements from airborne LiDAR data. IEEE Transations on, vol.41, $n^{\circ} .4$, p. $872-882,2003$.

\section{Biografia do autor principal}

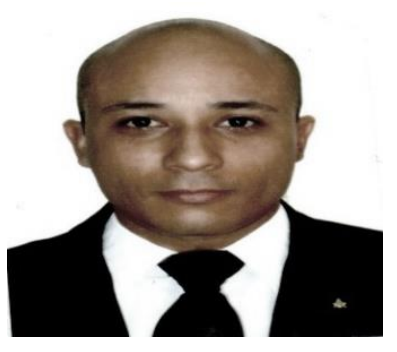

José Roberto Berithe Pedrosa, nascido em 9 de junho de 1982 na cidade de São Paulo. Possui curso técnico em eletrônica na Escola Técnica Federal de São Paulo (ETFSP) em 2000, formou-se na Academia Militar das Agulhas Negras (AMAN) em 2007, e graduou-se no Instituto Militar de Engenharia (IME) em 2013, no curso de Engenharia Cartográfica. Trabalhou no Projeto Radiografia da Amazônia como gerente operacional, na região da Amazônia Legal, e atualmente chefia a Divisão de Geoinformação no $2^{\circ}$ Centro de Geoinformação, quartel em Brasília-DF.

Esta obra está licenciada com uma Licença Creative Commons Atribuição 4.0 Internacional - CC BY. Esta licença permite que outros distribuam, remixem, adaptem e criem a partir do seu trabalho, mesmo para fins comerciais, desde que lhe atribuam o devido crédito pela criação original. 